\title{
Investigation on the effect of fractal soot aggregation on radiative transfer in homogeneous gas-soot mixture using full-spectrum k-distribution method
}

\author{
Zhenzong HE*, Dong LIANG*, Junkui MAO* and Xingsi HAN* \\ *Aero-engine Thermal Environment and Structure Key Laboratory of Ministry of Industry and Information Technology, Nanjing University \\ of Aeronautics and Astronautics, No. 29, Yudao Street, Nanjing, Jiangsu province, 210016, PR China \\ E-mail:mjkpe@nuaa.edu.cn
}

Received: 4 December 2018; Revised: 23 December 2018; Accepted: 6 January 2019

\begin{abstract}
The full-spectrum k-distribution (FSK) method and Generalized Multiparticle Mie-solution (GMM) method are applied to study the influences of soot aggregation on the radiative heat transfer in homogeneous gas-soot mixtures. The radiative transfer equation is solved by the finite volume method. The effect of soot aggregates with different fractal dimensions, monomer radiuses and the number of monomers on distributions of radiative heat flux and radiative heat source in the gas-soot mixture are studied, and the results show that increasing the fractal dimension, monomer radiuses, or decreasing the number of monomers will result in larger deviation between local radiative heat flux and radiative heat source of gas-soot mixture with soot aggregates and those with single soot particles. As a whole, the soot aggregation has a significant impact on the radiative heat transfer in the homogeneous gas-soot mixtures, and the effect of soot aggregation should better be considered as much as possible to obtain more accurate calculated results.
\end{abstract}

Keywords : Gas-soot mixture, Fractal aggregate, Full-spectrum k-distribution, Radiative heat transfer, Generalized Multiparticle Mie-solution method

\section{Introduction}

The oxy-fuel combustion usually contains multiple complex heat transfer mechanisms, and the thermal radiation is regarded as one of the principal mode of heat transfer in the flame. Dannecker et. al. (Dannecker, et al., 2007) studied the wall heat load at the liner structure of a model gas turbine combustion chamber and pointed out that the radiative heat transfer contributed to $20-30 \%$ of the total wall heat flux. Generally, the combustion products that result in the change of the radiative heat transfer in the oxy-fuel combustion flame include a large amount of gaseous species, e.g. $\mathrm{CO}_{2}$ and $\mathrm{H}_{2} \mathrm{O}$, which also exist in conventional air combustion, and the increasing concentration of particulate matters, e.g. soot (Guo, et al., 2018; Kangwanpongpan, et al., 2012; Li, et al., 2018). Therefore, to evaluate the heat transfer process of the oxy-fuel combustion flame accurately, the radiative properties of gases and soot in the flame should be studied carefully.

Generally, for the gas scattering is ignored due to its small scattering coefficient, the absorption and emitting properties are only considered. The common numerical calculation models used to predict the gas absorption and emitting properties contain Line-By-Line (LBL) model, Band models and Global models (Modest, 2013). The LBL model based on high-resolution spectroscopic databases, e.g. CDSD (Tashkun, et al., 2003) and HITEMP (Rothman, et al., 2010) can most accurately predict the radiative properties in the participating media, but are not mathematical trackable for requiring large computational effort. The Band model is an approximate method which employs the average values of gas radiative properties of the spectral band interval to replace the real value of that interval. Strictly, the Band model is a kind of semi-empirical formula, and the radiative property parameters are hypothetical and simplified. Unlike the LBL model and Band model, the Global model can not only ensure sufficient calculation precision but also be coupled with the radiative transfer equation (RTE) in differential form, which means various 
techniques, e.g. discrete ordinates method (DOM), Monte-Carlo method (MCM) and finite volume method (FVM), can be used (Chu, et al., 2014; Li, et al., 2018). The common Global models are the weighted-sum-of-gray-gases (WSGG) model, the full-spectrum k-distribution (FSK) model and spectral-line-based weighted-sum-of-gray-gases (SLW) model, etc. Among these models, the FSK model, based on the reordering concept and originally introduced by Modest (Modest, 2013), has attracted widespread attentions for its efficiency and accuracy in studying the radiative heat transfer in the non-gray participating media. Especially, by using the high-accuracy Gauss quadrature, the FSK model that needs integral over the spectrum can reduce the number of required radiative transfer equation (RTE) evaluations from over one million to around ten without losing spectral line information. Recently, the FSK model was extended to a gas and particle mixture and obtained satisfactory results (Wang, et al., 2016).

Unlike the gas radiation, the particle radiative properties have significant scattering properties more than absorption and emitting properties. Generally, except for complex refractive indices denoting the absorption and scattering abilities of radiation energy, the geometric features of particle also affect radiative properties of particle, especially for those of particle aggregates (Kholghy, et al., 2017). Recently, considerable numerical calculation methods have been introduced to predict heat radiative properties of particle aggregates, e.g. Generalized Multiparticle Mie-solution (GMM) method (Xu and Khlebtsov, 2003), superposition T-matrix method (Mishchenko, et al., 2002). Liu et. al. (Liu, et al., 2008) employed the superposition T-matrix method to study the radiative properties of soot fractal aggregates, and found that changing the fractal dimension of aggregates would result in significant change of the radiative properties of soot although the monomer radius and number unchanged. Li et. al. (Li, et al., 2010) compared the radiative properties of soot fractal aggregates with those of four equivalent-spherical approximations using the GMM method, and found that all these approximations gave rise to substantial errors, which means the effect of soot aggregation on radiative properties should be studied carefully and cannot be replaced by other approximate models.

In present study, the effects of radiative properties of soot aggregates on radiative heat transfer in gas-soot mixed medium are studied. The spectral radiative properties of soot aggregates are predicted by the GMM method, the spectral radiative properties of gas are obtained from HITEMP2010 database, and the non-gray radiative properties of gas-soot mixtures are studied by the FSK model. The remainder of this research is organized as follows. First, the radiative properties of the gas and soot aggregates are studied, and the principle of the FSK model is introduced. Then, the effect of aggregate geometrical parameters, e.g. fractal dimension, primary monomer radius and number of monomer in aggregates, on the radiative heat transfer in gas-soot mixtures are studied. Finally, the main conclusions and perspectives are provided.

\section{Theoretical Background}

\subsection{Gas radiative properties}
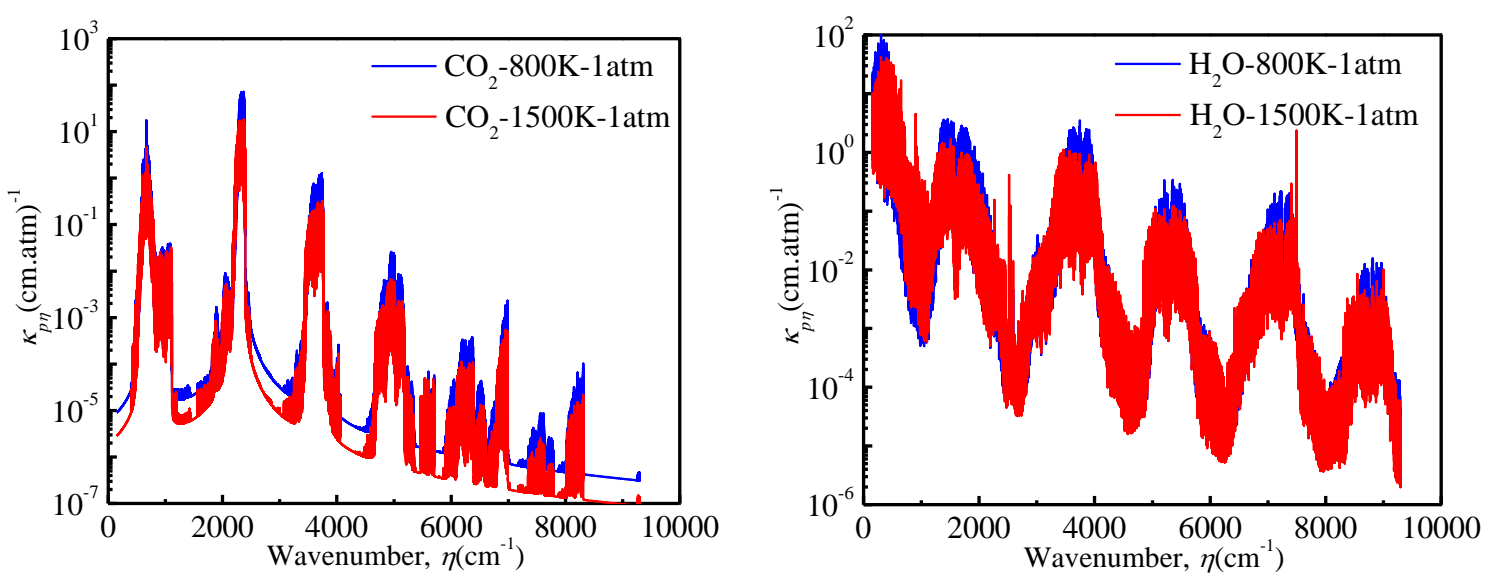

Fig. 1 Spectral pressure absorption coefficients for $\mathrm{H}_{2} \mathrm{O}$ and $\mathrm{CO}_{2}$ obtained from HITEMP2010 at $800 \mathrm{~K}$ and $1500 \mathrm{~K}$.

Generally, gas radiative properties, dependent on environmental temperature, pressure, gas concentration, incident wavenumber, denote the abilities to absorb external radiative energy and emit radiative energy. So, to simulate the radiative heat transfer in participating gaseous medium accurately, one important work is to establish high-resolution spectral database that can provide spectroscopic parameters to generate the transition lines. Nowadays, the most widely 
used databases are the HITRAN database and HITEMP database. The HITRAN database was built at a reference temperature of $296 \mathrm{~K}$ for atmospheric applications, while the HITEMP database was established for high temperature applications, and contained combustion gases, such as $\mathrm{CO}_{2}, \mathrm{H}_{2} \mathrm{O}, \mathrm{CO}$ and $\mathrm{OH}$. Comparing with other available databases, the updated HITEMP2010 database has been proved to be the most accurate one in studying the radiative heat transfer in the participating gaseous medium (Dorigon, et al., 2013). Figure 1 depicts the spectral pressure absorption coefficients for $\mathrm{H}_{2} \mathrm{O}$ and $\mathrm{CO}_{2}$ obtained from HITEMP2010 at $800 \mathrm{~K}$ and $1500 \mathrm{~K}$, respectively.

\subsection{Radiative properties of soot aggregates}

Nowadays, there are several kinetic growth models used to simulate the fractal growth process of small particles to form large aggregates, such as the diffusion-limited aggregation (DLA), ballistic aggregation (BA), reaction-limited aggregation (RLA), and cluster-cluster aggregation (CCA) models (Gouyet and Mandelbrot, 1996). In the present work, the CCA model, developed by Mackowski (Mackowski, 2006) and widely used in studying the aggregation of soot, aerosols and other particles (Li, et al., 2018; Liu, Mishchenko and Patrick Arnott, 2008; Ren, et al., 2018), is proposed to create the aggregates. It assumes that many particles diffuse randomly at the same time. If the particles touch one another, they adhere together and form aggregates. All the particles and aggregates move randomly and touch other particles or aggregates until all of them form one fractal aggregate (Meakin, et al., 1984). Thus, the arrangement of particles in the CCA model is random and the core particle does not exist in the aggregate as in the DLA model. According to the fractal theory, the morphology and construction of the aggregate can be described as (Sorensen, et al., 2018):

$$
\begin{aligned}
& N_{\mathrm{s}}=k_{\mathrm{f}}\left(\frac{R_{\mathrm{g}}}{a}\right)^{D_{\mathrm{f}}} \\
& R_{\mathrm{g}}^{2}=\frac{1}{N_{\mathrm{s}}} \sum_{i=1}^{N_{\mathrm{s}}} r_{i}^{2}
\end{aligned}
$$

where $N_{\mathrm{s}}$ is the total number of primary monomers in the aggregate; $a$ is the mean radius of the monomers; $k_{\mathrm{f}}$ is the fractal prefactor; $D_{\mathrm{f}}$ is the fractal dimension; $R_{\mathrm{g}}$ denotes a root mean square radius that quantifies the overall size of the aggregate and is called the gyration radius; $r_{i}$ is the distance from the $i$ th sphere to the center of the aggregate mass. The relationship between the gyration radius $R_{\mathrm{g}}$ and the number of monomers $N_{\mathrm{s}}$ in the aggregates can thus be connected by the fractal dimension $D_{\mathrm{f}}$ and fractal prefactor $k_{\mathrm{f}}$. Liu et al. (Liu and Mishchenko, 2005) studied the contribution of the aggregate geometrical parameters ( $D_{\mathrm{f}}, k_{\mathrm{f}}, a$, and $N_{\mathrm{s}}$ ) to scattering and absorption properties of aggregates, and revealed that the effects of $k_{\mathrm{f}}$ variation on phase function, the ratios of scattering matrix elements, and aggregate morphology were similar but visibly weak. So, to simplify the calculation, the radiative properties of soot aggregates are main dependent on the fractal dimension $D_{\mathrm{f}}$, if the total number of monomers $N_{\mathrm{s}}$ and the mean radius of the primary particles $a$ are fixed. Figure 2 shows different soot aggregates simulated by CCA model with the same $D_{\mathrm{f}}, N_{\mathrm{s}}$ and $a$. It is obvious that although these aggregates have the same fractal dimension, monomer radius and total number of monomers, the arrangement modes are different from each other.

Table 1 Aggregate geometrical parameters for the aggregates in Fig. 3

\begin{tabular}{c|c|c|c|c|c|c|c|c|c|c}
\hline \hline \multicolumn{2}{c|}{$D_{\mathrm{f}}$} & 1.20 & 1.40 & 1.60 & 1.80 & 2.00 & 2.20 & 2.40 & 2.60 & 2.80 \\
\hline \multirow{2}{*}{$N_{s}=20$} & $k_{\mathrm{f}}$ & 2.192 & 1.910 & 1.542 & 1.499 & 1.502 & 1.522 & 1.471 & 1.283 & 1.187 \\
& $R_{\mathrm{g}} / a$ & 6.312 & 5.353 & 4.961 & 4.218 & 3.649 & 3.224 & 2.967 & 2.876 & 2.742 \\
\hline
\end{tabular}

Figure 3 exhibits the aggregates generated for the various fractal dimensions simulated by the CCA model. The number of monomers in the aggregates is fixed at 20 , and the radius of primary monomers is fixed at $0.1 \mu \mathrm{m}$. The prefactors and gyration radiuses are pre-specified according to Table 1. It is obvious that the aggregates with larger fractal dimension show more compactly. Figure 4 shows the comparison between the laboratory microwave scattering measurements $(\mathrm{Xu}$ and Khlebtsov, 2003) and theoretical predictions by GMM method and superposition T-matrix 
method, respectively, for the angular distributions of dimensionless polarized scattering intensities $i_{11}$ and $i_{22}$. The incident light with wavelength $\lambda=3.997 \mu \mathrm{m}$ is along the centre line of the 2-particles aggregate. The size parameter of the monomer is 7.86, and the complex refractive index of primary monomer is set as 2.5155-0.0213 $i$. It is obvious that both the GMM method and superposition T-matrix method show satisfactory simulated precision, especially the GMM method. Therefore, the following studies about the radiative properties of aggregates are based on GMM method.

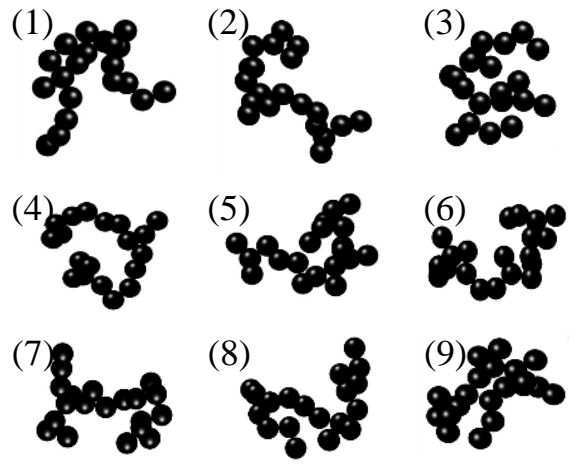

Fig. 2 Fractal aggregates with $N_{\mathrm{s}}=20, D_{\mathrm{f}}=1.5$ and $a=0.1$ $\mu \mathrm{m}$.

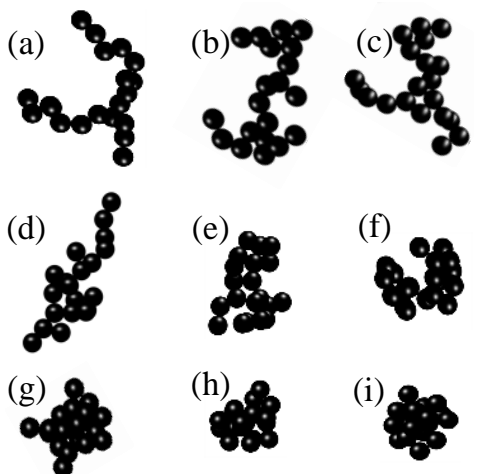

Fig. 3 Fractal aggregates with $N_{\mathrm{s}}=20, a=0.1 \mu \mathrm{m}$ and characterized by different fractal dimension values $D_{\mathrm{f}}$ according to Table 1 . (a)-(i) $D_{\mathrm{f}}=1.2$, 1.4, 1.6, 1.8, 2.0, 2.2, 2.4, 2.6, 2.8 .
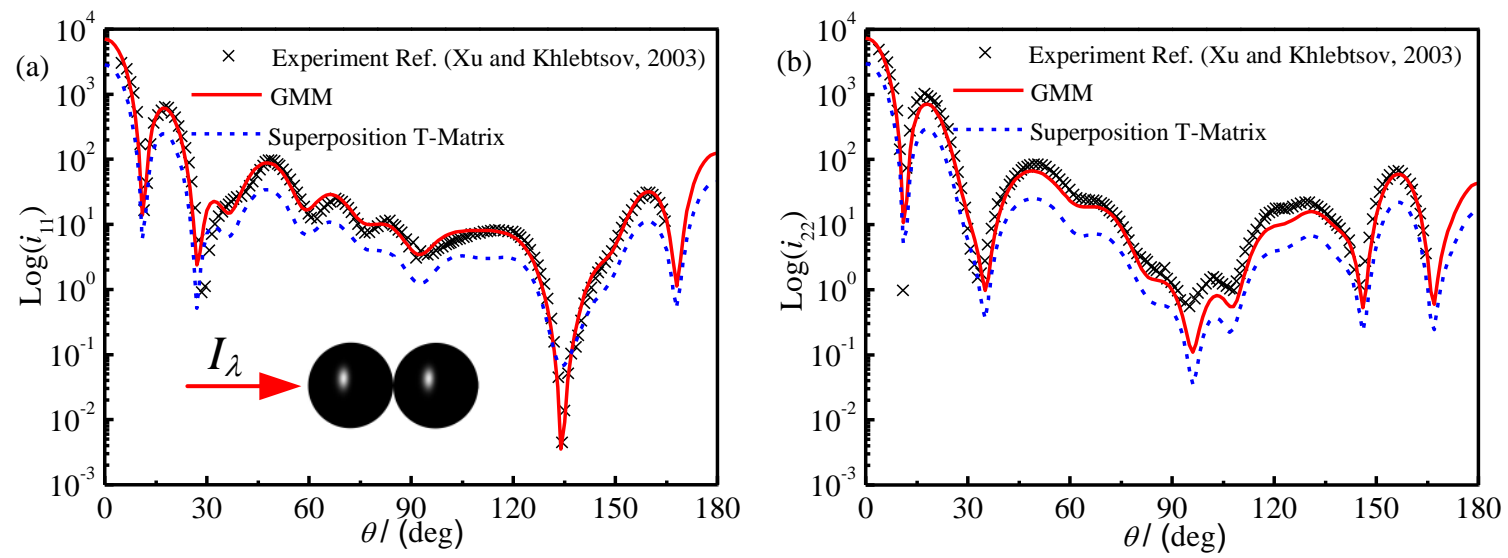

Fig. 4 Comparison between theoretical predictions and laboratory microwave scattering measurements for the angular distributions of $i_{11}$ and $i_{22}$.

Table 2 Radiative properties for soot aggregates shown in Fig. 2 at wavelength $\lambda=0.83 \mu \mathrm{m}$

\begin{tabular}{c|c|c|c|c|c}
\hline \hline Number & $\left\langle C_{\mathrm{ext}}\right\rangle /\left(\mu \mathrm{m}^{2}\right)$ & $\left\langle C_{\mathrm{abs}}\right\rangle /\left(\mu \mathrm{m}^{2}\right)$ & $\left\langle C_{\mathrm{sca}}\right\rangle /\left(\mu \mathrm{m}^{2}\right)$ & $\omega_{\mathrm{alb}}$ & $\langle\cos \theta\rangle$ \\
\hline 1 & 0.796717 & 0.481962 & 0.314755 & 0.395065 & 0.666133 \\
2 & 0.796971 & 0.476176 & 0.320795 & 0.402518 & 0.674779 \\
3 & 0.799900 & 0.482028 & 0.317872 & 0.397389 & 0.653647 \\
4 & 0.800626 & 0.479543 & 0.321083 & 0.401040 & 0.651258 \\
5 & 0.797566 & 0.479007 & 0.318559 & 0.399414 & 0.680386 \\
6 & 0.800307 & 0.482457 & 0.317850 & 0.397161 & 0.640504 \\
7 & 0.796746 & 0.474328 & 0.322417 & 0.404668 & 0.670683 \\
8 & 0.800474 & 0.480697 & 0.319777 & 0.399485 & 0.656257 \\
9 & 0.800482 & 0.477973 & 0.322509 & 0.402894 & 0.648357 \\
Average value & 0.798865 & 0.479352 & 0.319513 & 0.399959 & 0.660223 \\
Standard error & 0.001797 & 0.002802 & 0.002515 & 0.003111 & 0.013378 \\
\hline
\end{tabular}



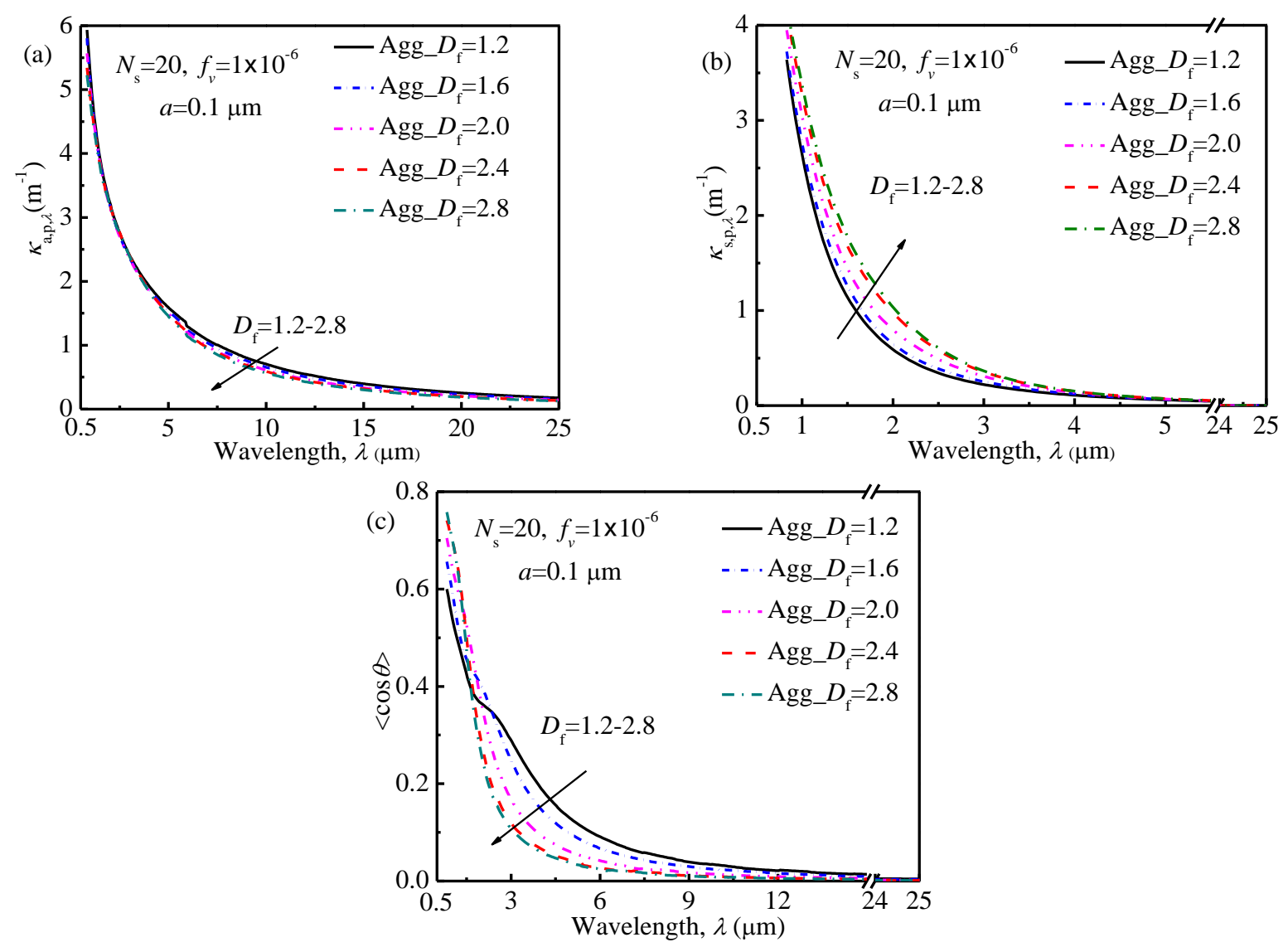

Fig. 5 Spectral radiative properties of soot aggregates as functions of fractal dimensions $D_{\mathrm{f}}$ : (a) scattering coefficient, (b) absorption coefficient, (c) asymmetry factor.

According to Hofgren and Sundén's work (Hofgren and Sundén, 2015), the wavelength range in studying the radiative properties of soot is set as $0.83-25.0 \mu \mathrm{m}$, and the spectral complex refraction indices are generally modeled using the correlation developed by Chang and Charalampopoulos (Chang and Charalampopoulos, 1990):

$$
\begin{aligned}
& n_{\lambda}=1.811+0.1263 \ln \lambda+0.027 \ln ^{2} \lambda+0.0417 \ln ^{3} \lambda \\
& k_{\lambda}=0.5821+0.1213 \ln \lambda+0.2309 \ln ^{2} \lambda-0.01 \ln ^{3} \lambda
\end{aligned}
$$

where $n_{\lambda}$ and $k_{\lambda}$ denote the real part and the imaginary part of the complex refractive indices, respectively, which are the single-valued functions of wavelengths. Table 2 illustrates the radiative properties (i.e. absorption cross section $\left\langle C_{\mathrm{abs}}\right\rangle$, scattering cross section $\left\langle C_{\mathrm{sca}}\right\rangle$, extinction cross section $\left\langle C_{\mathrm{ext}}\right\rangle$, scattering albedo $\omega_{\mathrm{alb}}$ and asymmetry factor $\langle\cos \theta\rangle$ ) of soot aggregates showed in Fig. 2 at wavelength $\lambda=0.83 \mu \mathrm{m}$. It is obvious that there is almost no difference between the spectral radiative properties of soot aggregates with different arrangements, while the fractal dimension $D_{\mathrm{f}}$, monomer radius $a$ and total number of monomers $N_{\mathrm{s}}$ are kept the same. The absorption coefficient $\kappa_{\mathrm{a}, \mathrm{p}}$ and scattering coefficient $\kappa_{\mathrm{s}, \mathrm{p}}$ of the soot dispersion medium can be expressed as (Guo, Hu, Luo, Li and Liu, 2018):

$$
\kappa_{\mathrm{a}, \mathrm{p}}=\frac{\left\langle C_{\mathrm{abs}}\right\rangle \cdot f_{\mathrm{v}}}{N_{s} \times 4 \pi a^{3} / 3}, \quad \kappa_{\mathrm{s}, \mathrm{p}}=\frac{\left\langle C_{\mathrm{sca}}\right\rangle \cdot f_{\mathrm{v}}}{N_{s} \times 4 \pi a^{3} / 3}
$$

where $f_{\mathrm{v}}$ denotes the total particle volume fraction. Figure 5 illustrates the spectral radiative properties of the soot dispersed media with aggregates showed in Fig. 3 within the wavelengths $[0.83,25] \mu \mathrm{m}$. The total particle volume fraction $f_{\mathrm{v}}$ is set as $1 \times 10^{-6}$, and the radius of the primary monomers is set as $0.1 \mu \mathrm{m}$. It can be found that increasing fractal dimensions of aggregates will result in the increasing of spectral scattering coefficients, while the spectral absorption coefficients will decline. The influence on the asymmetry factor is slightly complicated, and the values of 
asymmetry factor reduces. The likely explanation may be that for soot aggregates with larger fractal dimension, e.g. the $i$ th aggregate in Fig. 3, less surface of the aggregate directly exposes to the incident radiation, which will result in the decreasing of the absorption cross section. On the other hand, the superposition of the scattering field of monomers in the aggregates will enhance the scattering interaction between the primary monomers as fractals age.

\subsection{Radiative transfer equation (RTE)}

For an absorbing, emitting, and scattering gas-particle mixture, the change of radiation intensity is obtained by the sum of the emission, absorption, scattering away from the direction $\overrightarrow{\boldsymbol{s}}$, and scattering into the direction $\overrightarrow{\boldsymbol{s}}$. The gas scattering is ignored normally, due to its small scattering coefficient. Moreover, in order to make the problem mathematically trackable, the temperature of particles is assumed the same as the gas. Therefore, the radiative transfer equation in spectral form can be derived as (Modest, 2013; Qi, et al., 2019):

$$
\frac{\mathrm{d} I_{\eta}(z, \vec{s})}{\mathrm{d} z}=-\left(\kappa_{\mathrm{a}, \eta}+\kappa_{\mathrm{s}, \eta}\right) I_{\eta}(z, \vec{s})+\kappa_{\mathrm{a}, \eta} I_{\mathrm{b}}(z)+\frac{\kappa_{\mathrm{s}, \eta}}{4 \pi} \int_{4 \pi} I_{\eta}(z, \vec{s}) \Phi\left(\vec{s}^{\prime}, \vec{s}\right) \mathrm{d} \Omega^{\prime}
$$

where $\eta$ denotes the wavenumber; $\kappa_{\mathrm{a}, \eta}, \kappa_{\mathrm{s}, \eta}$ and $\Phi\left(\vec{s}^{\prime}, \vec{s}\right)$ are the spectral absorption coefficient, scattering coefficient and scattering phase function of the participating medium. For the gas-particle mixtures, there are $\kappa_{\mathrm{a}, \eta}=\kappa_{\mathrm{g}, \eta}+\kappa_{\mathrm{a}, \mathrm{p}, \eta}$ and $\kappa_{\mathrm{s}, \eta}=\kappa_{\mathrm{s}, \mathrm{p}, \eta} . \kappa_{\mathrm{g}, \eta}$ is the gas spectral absorption coefficient. $\kappa_{\mathrm{a}, \mathrm{p}, \eta}$ and $\kappa_{\mathrm{s}, \mathrm{p}, \eta}$ are the spectral absorption coefficient and spectral scattering coefficient of particles.

Considering black walls with known temperatures, the boundary conditions for a diffusely emitting and reflecting surface can be expressed as follow (Sun, et al., 2016):

$$
I_{\eta}(z, \vec{s})=\varepsilon_{w} I_{\mathrm{b}, \eta}(z, \vec{s})+\frac{1-\varepsilon_{w}}{\pi} \int_{\vec{s}^{\prime} \cdot \vec{n}<0} I_{\eta}\left(z, \vec{s}^{\prime}\right)\left|\vec{s}^{\prime} \cdot \vec{n}\right| \mathrm{d} \Omega^{\prime}
$$

where $\varepsilon_{w}$ denotes the emitting rate of the boundary with $\varepsilon_{w}=1$ for the black wall; $\vec{n}$ is the outer normal direction of the wall.

\subsection{Full-spectrum k-distribution (FSK) method}

Usually, the absorption coefficients of gas fluctuate seriously with wavenumbers (See Fig.1). To make the absorption coefficients trackable, the full-spectrum k-distribution model, a kind of probability distribution function, was introduced to rearrange and calculate spectral absorption coefficients of gas according to the probability of the occurrence of absorption coefficients, if the Planck function changes only slightly over a small spectral interval. However, the Planck function varies significantly across the spectrum. So, the blackbody intensity should be considered in the full spectrum case and the blackbody intensity weighted full-spectrum k-distribution can be defined as (Modest, 2013; Wang, Modest and He, 2016):

$$
f(T, k)=\frac{1}{I_{b}(T)} \int_{0}^{\infty} I_{b, \eta}(T) \delta\left(k-\kappa_{\eta}\right) \mathrm{d} \eta
$$

where $\delta\left(k-\kappa_{\eta}\right)$ is the Dirac-delta function; $\kappa_{\eta}$ denotes the spectral radiative property parameters calculated from a spectroscopic database, such as absorption coefficient and scattering coefficient; $I_{b}(T)$ and $I_{b, \eta}(T)$ denote total blackbody intensity obtained integrating the Plank function over the entire spectrum and spectral blackbody intensity at wavenumber $\eta$, respectively. The corresponding cumulative full-spectrum $k$-distribution, which denotes the fraction of the spectrum whose radiative property parameters lie below the value $k$, can be expressed as:

$$
g(T, k)=\int_{0}^{k} f\left(T, k^{\prime}\right) \mathrm{d} k^{\prime}
$$

Therefore, for a homogeneous gas-particle mixture, the RTE and boundary condition can be reordered in smoothly-varying $g$-space as: 


$$
\begin{aligned}
& \frac{\mathrm{d} I_{g}(z, \vec{s})}{\mathrm{d} z}=-\left(\kappa_{\mathrm{a}, g}+\kappa_{\mathrm{s}, g}\right) I_{g}(z, \vec{s})+\kappa_{\mathrm{a}, g} I_{\mathrm{b}}(z)+\frac{\kappa_{\mathrm{s}, g}}{4 \pi} \int_{4 \pi} I_{g}(z, \vec{s}) \Phi\left(\vec{s}^{\prime}, \vec{s}\right) \mathrm{d} \Omega^{\prime} \\
& I_{g}\left(z_{w}, \vec{s}\right)=\varepsilon_{w} a\left(T_{w}, T, g\right) I_{\mathrm{b}}\left(z_{w}, \vec{s}\right)+\frac{1-\varepsilon_{w}}{\pi} \int_{\vec{s}^{\prime} \cdot \vec{n}<0} I_{g}\left(z_{w}, \vec{s}^{\prime}\right)\left|\vec{s}^{\prime} \cdot \vec{n}\right| \mathrm{d} \Omega^{\prime}
\end{aligned}
$$

where $I_{g}$ denotes the radiative intensity being solved in $g$-space; $a\left(T_{w}, T, g\right)$ is a non-gray stretching factor accounting for the difference of wall temperature $T_{w}$ from the temperature $T$ in Planck function that is used to construct the $k$ - $g$ distribution. The mathematical expressions of $I_{g}$ and $a\left(T_{w}, T, g\right)$ are defined as:

$$
\begin{aligned}
& I_{g}=\int_{0}^{\infty} I_{\eta} \delta\left(k-\kappa_{\eta}\right) \mathrm{d} \eta / f(T, k) \\
& a\left(T_{w}, T, g\right)=\frac{f\left(T_{w}, k\right)}{f(T, k)}=\frac{d g_{w}\left(T_{w}, k\right)}{d g(T, k)}
\end{aligned}
$$

Then, with the spectral integration performed by using a high-accuracy Gauss quadrature, the number of required RTE evaluations can be greatly reduced without losing accuracy. The total radiative intensity for the entire spectrum can be evaluated through an $N$-point numerical quadrature:

$$
I=\int_{0}^{1} I_{g} \mathrm{~d} g=\sum_{i=1}^{N} w_{i} I_{g_{i}}
$$

where $w_{i}$ denotes the weight factors obtained from the Gauss quadrature; $I_{g_{i}}$ is the radiative intensity for quadrature point $g_{i}$. The incident radiation coming from all directions can be derived as (Trivic, 2014):

$$
G_{t o t}(z)=\int_{0}^{1} G_{g}(z) \mathrm{d} g=\sum_{i=1}^{N} w_{i} \int_{4 \pi} I_{g_{i}}(z, \vec{s}) \mathrm{d} \Omega
$$

The radiative heat flux $q_{t o t}$ and radiative heat source $\nabla \bullet q_{t o t}$ for all gas-particle mixtures are expressed as:

$$
\begin{aligned}
& q_{t o t}(z)=\int_{0}^{1} q_{g}(z) \mathrm{d} g=\int_{0}^{1} \int_{4 \pi} I_{g}(z, \vec{s})(\vec{s} \bullet \vec{n}) \mathrm{d} \Omega \mathrm{d} g=\sum_{i=1}^{N} w_{i} \int_{4 \pi} I_{g_{i}}(z, \vec{s})(\vec{s} \bullet \vec{n}) \mathrm{d} \Omega \\
& \nabla \bullet q_{t o t}(z)=\int_{0}^{1} \nabla \bullet q_{g}(z) \mathrm{d} g=\sum_{i=1}^{N} w_{i} \kappa_{g_{i}}(z)\left[4 \pi I_{\mathrm{b}}(z)-G_{g_{i}}(z)\right]
\end{aligned}
$$

where $\vec{n}$ is the direction vector. Figure 6 shows the calculated results of the distributions of radiative heat flux and radiative heat source in $1 \mathrm{D}$ medium mixed with $\mathrm{H}_{2} \mathrm{O}$ and $\mathrm{CO}_{2}$, and it is obvious that there is a reasonable agreement between the results calculated by the LBL-DOM in the Ref. (Guo, et al., 2015) and those by the FSK-FVM in the present study. 

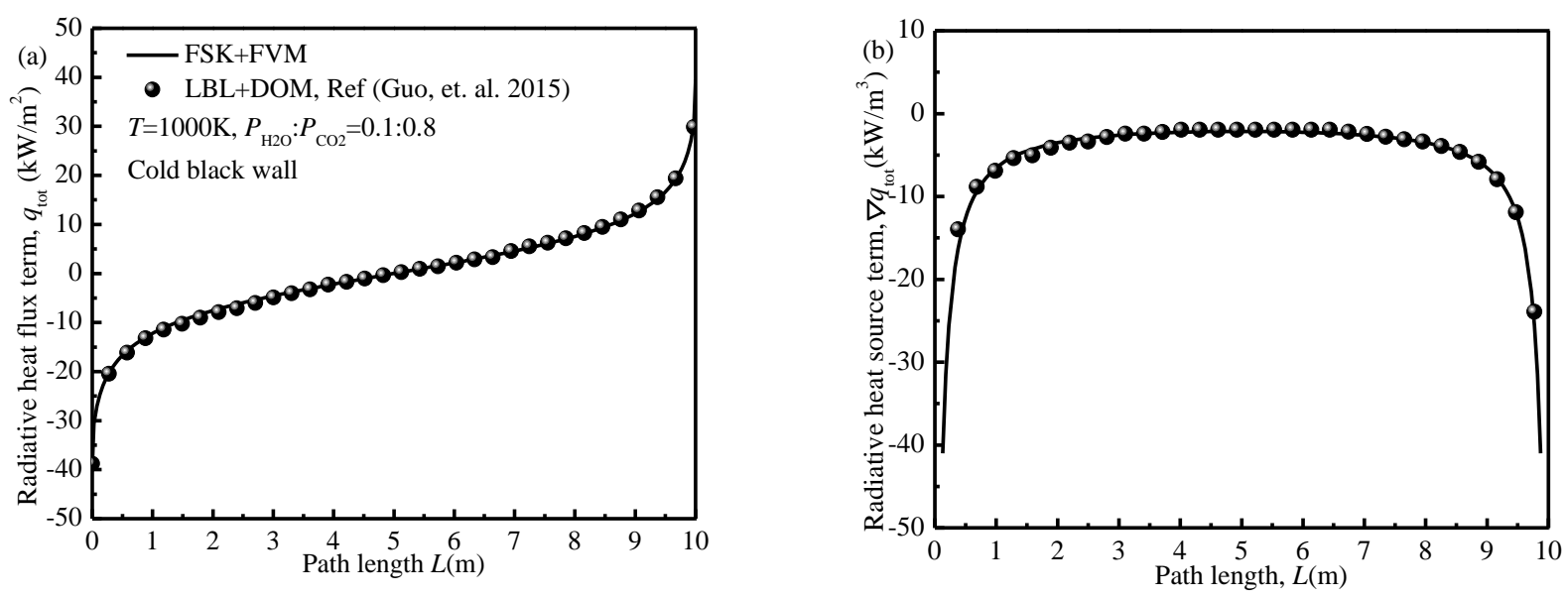

Fig. 6 Comparison between FSK-FVM and LBL-DOM available in Ref (Guo, Li, Huang, Liu and Zheng, 2015) in studying the radiative heat transfer in $1 \mathrm{D}$ gaseous mixtures

\section{Numerical simulation}

By applying the methodology mentioned above, the effects of fractal soot aggregation on radiative heat transfer in homogeneous gas-soot mixture are studied. Moreover, the relative errors of the radiative heat flux and radiative heat source in the gas-soot mixtures with single soot particles and those with soot aggregates are also studied. To facilitate the mathematical trackability, the following assumptions are made: 1) the temperature in the gas-soot mixture is assumed to be uniform, and the wall temperature is set as $0 \mathrm{~K}$; 2) the difference of the temperature between the gas components and soot is not considered; 3 ) the total soot volume fraction $f_{\mathrm{v}}$ is set as $1 \times 10^{-6}$, and the effect of soot on the volume of the gas-soot mixture is ignored for the small volume; 4) both the gaseous and soot components are assumed to be a uniform mixture; 5) the soot aggregates are assumed to be uniform-diameter just-touching primary monomers; 6) the concentration ratio of gaseous components are $\mathrm{CO}_{2}: \mathrm{H}_{2} \mathrm{O}: \mathrm{N}_{2}=1: 1: 8$, and the total pressure is set as 1 atm; 7) for the gas-soot mixtures with single soot particle, the independent scattering dominates and the scattering interaction between particles is ignored. Figure 7 shows the corresponding k-distributions of gaseous components at different temperatures. It can be found that increasing the concentration of $\mathrm{H}_{2} \mathrm{O}$ or decreasing the medium temperature will result in the increasing of gas absorption coefficients. Figure 8 depicts the $k$-distributions for the radiative properties of soot aggregates at $1500 \mathrm{~K}$, and the other properties of the aggregates are the same as those studied in Fig. 5.

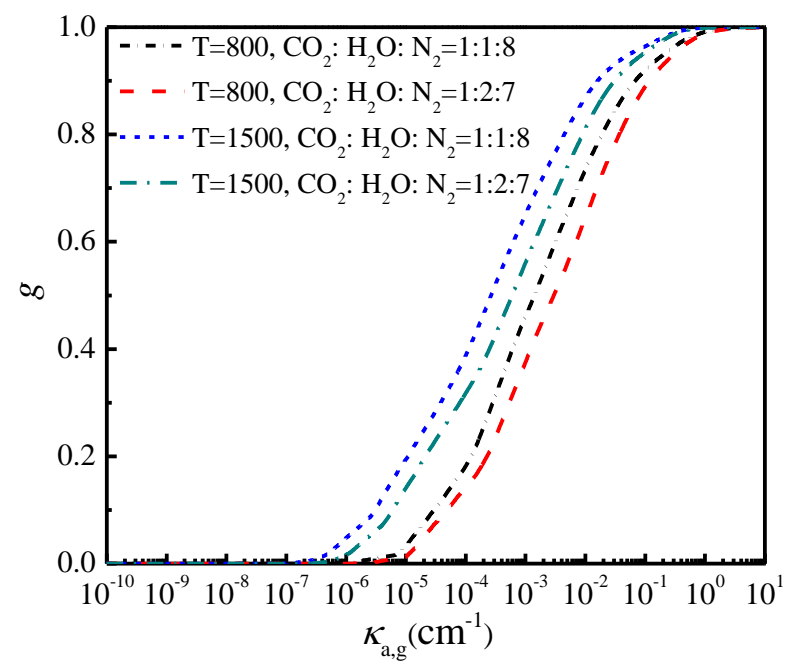

Fig. 7 The k-distributions for gaseous components at different temperatures calculated from HITEMP2010 database 

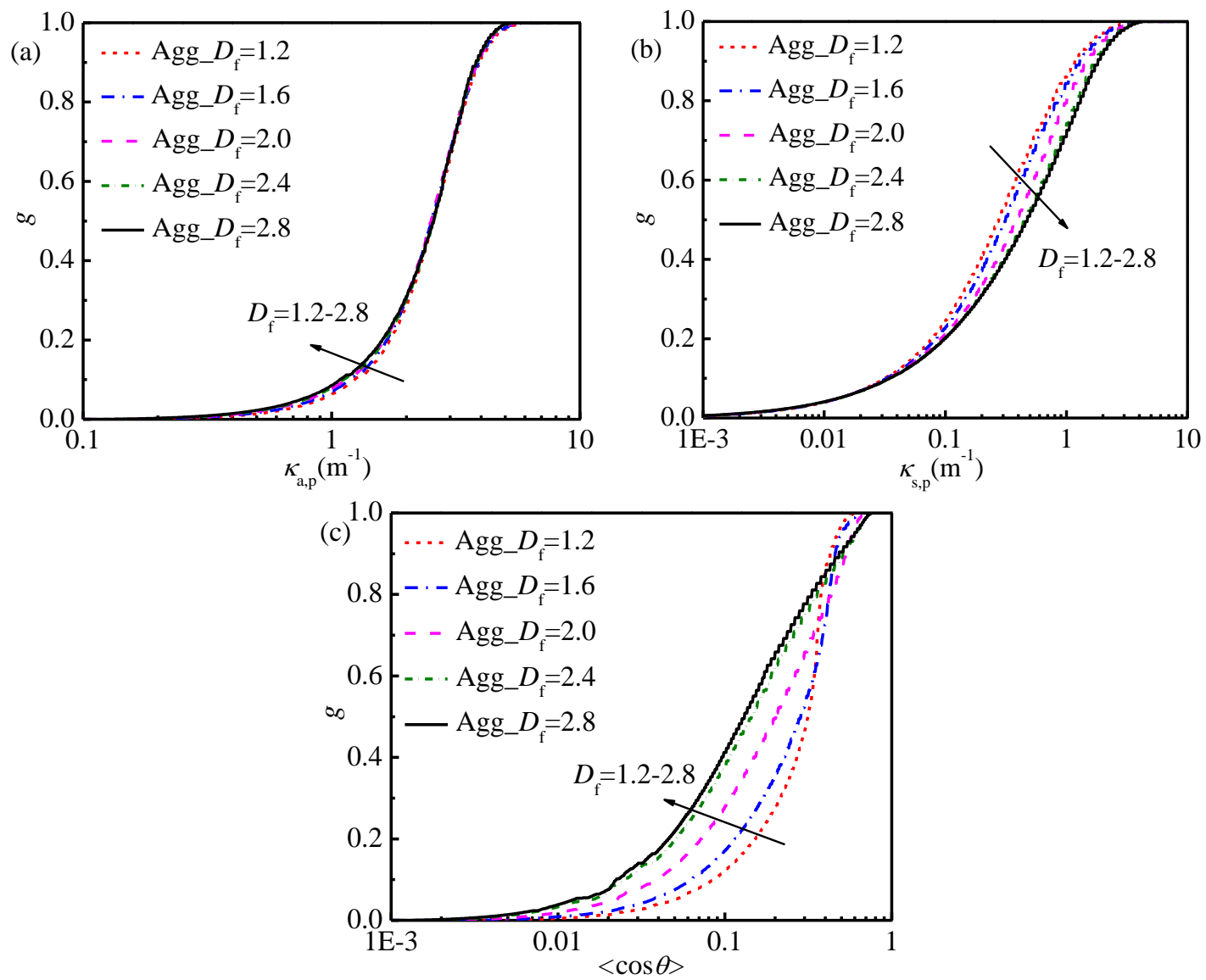

Fig. 8 The k-distributions for the radiative properties of soot aggregates with different fractal dimensions $D_{\mathrm{f}}$ at $1500 \mathrm{~K}$

\subsection{Effect of fractal dimensions on the radiative heat transfer properties in the gas-soot mixtures}

Figure 9 shows the calculated results of radiative heat flux and radiative heat source in the gas-soot mixtures consisted of soot aggregates with different fractal dimensions $\left(D_{\mathrm{f}}=1.2,1.6,2.0,2.4\right.$ and 2.8) and those consisted of single soot particles, respectively. The radius of the monomers is set as $0.1 \mu \mathrm{m}$, the temperature in the gas-soot mixture is set as $1500 \mathrm{~K}$, and the total number of primary monomers in the aggregates is set as 20 . The results show that ignoring the effect of soot aggregation will result in the increasing of the absolute value of radiative heat flux and overpredicting the value of radiative heat flux. While increasing the fractal dimensions of the aggregates will cause the relative errors of radiative heat flux to be larger, which means for soot aggregates with larger fractal dimensions, ignoring the effect of soot aggregation will result in larger deviation. The likely explanation may be the same as those mentioned in studying the results depicted in Fig. 5. Similar conclusion can be obtained in studying the radiative heat source.
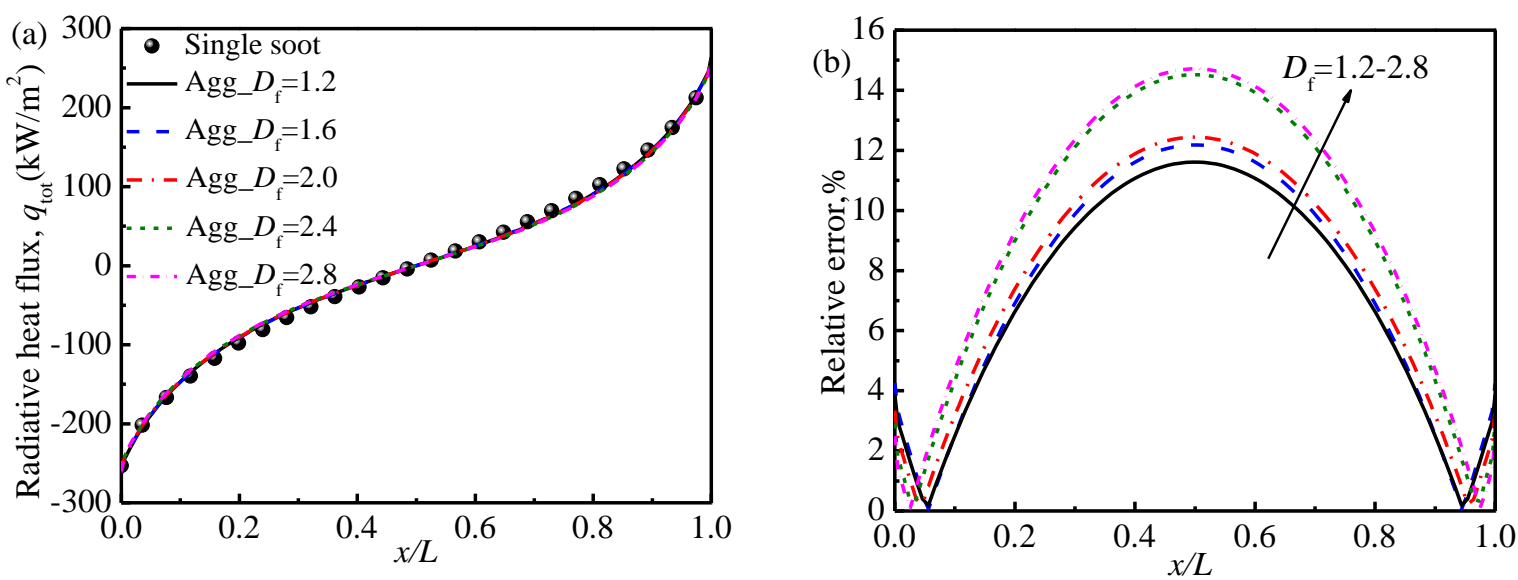

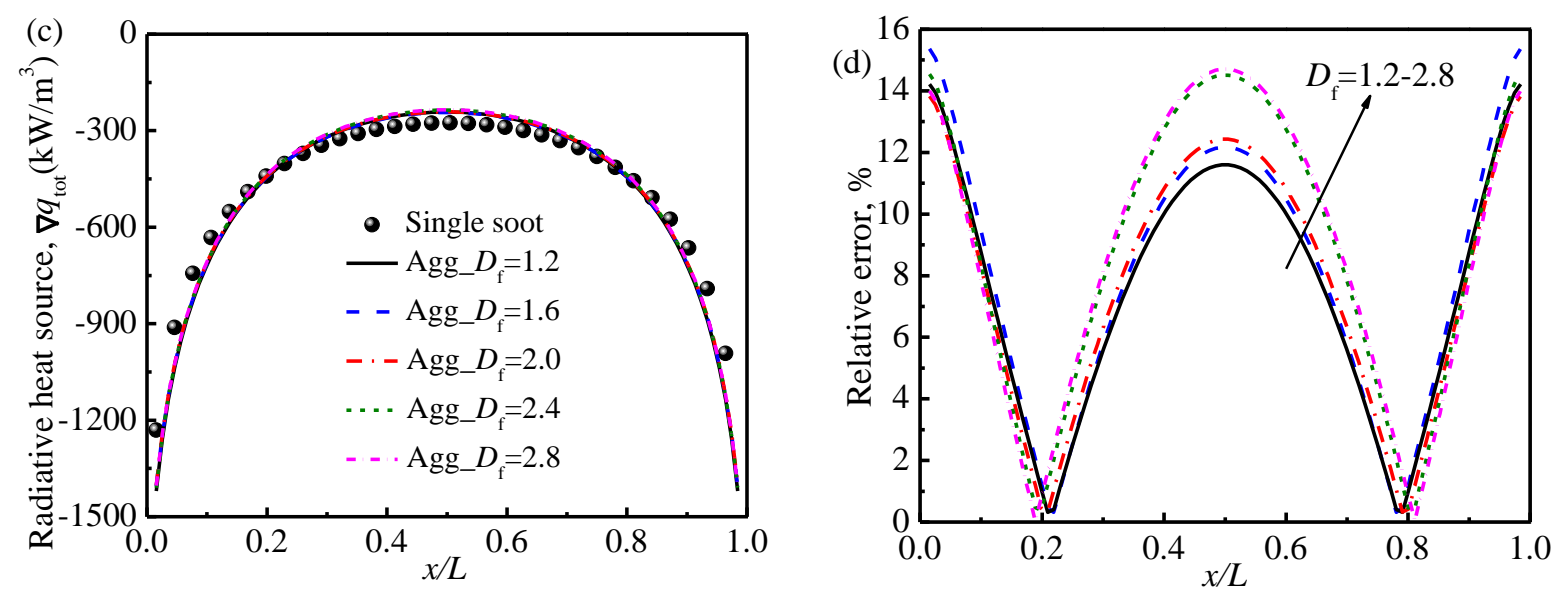

Fig. 9 Radiative heat transfer properties in the gas-soot mixture with different fractal dimensions $D_{\mathrm{f}}$ : (a) and (b) denoting the value and corresponding relative error of radiative heat flux; (c) and (d) denoting those for radiative heat source.

\subsection{Effect of primary monomer radius on the radiative heat transfer properties in the gas-soot mixtures}
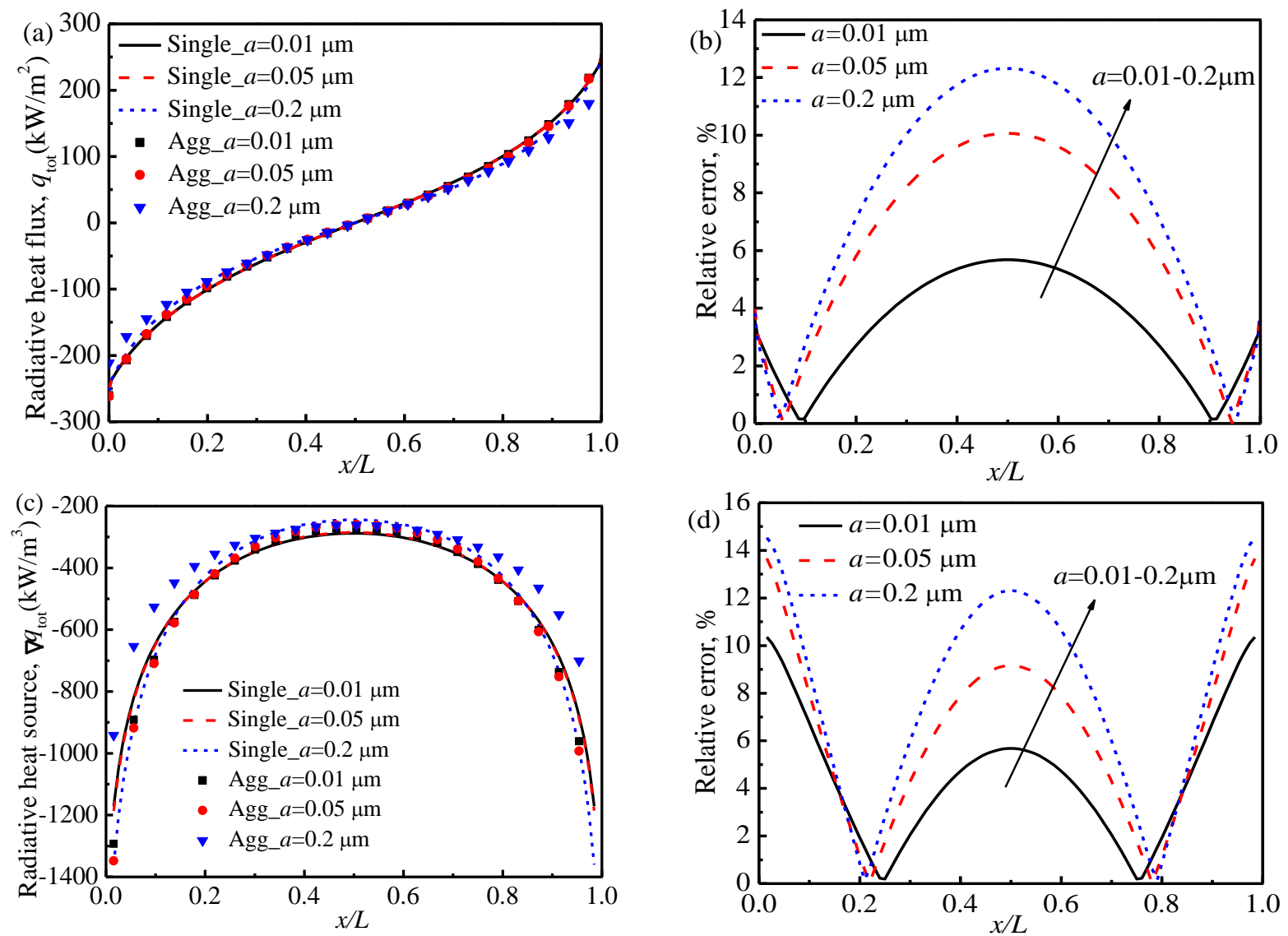

Fig. 10 Radiative heat transfer properties in the gas-soot mixture with different monomer radius a: (a) and (b) denoting the value and corresponding relative error of radiative heat flux; (c) and (d) denoting those for radiative heat source.

Figure 10 shows the calculated results of radiative heat flux and radiative heat source in the gas-soot mixture consisted of soot aggregates with different monomer radiuses $(a=0.01 \mu \mathrm{m}, 0.05 \mu \mathrm{m}$ and $0.2 \mu \mathrm{m})$ and those consisted of single soot particles, respectively. The fractal dimension $D_{\mathrm{f}}$ and the total number of monomers $N_{\mathrm{s}}$ in the aggregates are set as 1.8 and 20, respectively. The temperature in the gas-soot mixture is set as $1500 \mathrm{~K}$. It is obvious that if ignoring 
the effect of soot aggregation, the absolute value of local radiative heat flux also becomes larger. When increasing the radius of primary monomers in the aggregates, the relative errors of radiative heat flux become larger, which means for soot aggregates with larger size monomers, ignoring the effect of soot aggregation will result in larger deviation. The likely explanation may be that according to the Rayleigh scattering theory, the scattering effect is weak and the radiative interaction between monomers in the aggregates can be ignored for primary monomers with small size parameter (e.g. $\chi \ll 1$ ), while increasing the monomer size, the scattering effect enhances and the radiative interaction should be considered.

\subsection{Effect of monomers number on the radiative heat transfer properties in the gas-soot mixtures}

Figure 11 shows the calculated results of radiative heat flux and radiative heat source in the gas-soot mixtures consisted of soot aggregates with different number of monomers $\left(N_{\mathrm{s}}=10,20\right.$ and 30) and those consisted of single soot particles, respectively. The fractal dimension $D_{\mathrm{f}}$ is set as 1.8 , the temperature in the gas-soot mixture is set as $1500 \mathrm{~K}$, and the radius of primary monomers is set as $0.1 \mu \mathrm{m}$. It can be found that decreasing the number of primary monomers in the aggregates will result in the increasing of the relative errors of radiative heat flux and radiative heat source. The likely explanation may be that although more particles aggregating and blocking from each other will result in the declining of absorption coefficient, the scattering interaction between particles will be enhanced simultaneously. So, the integrated effects may lead to the phenomena mentioned above.
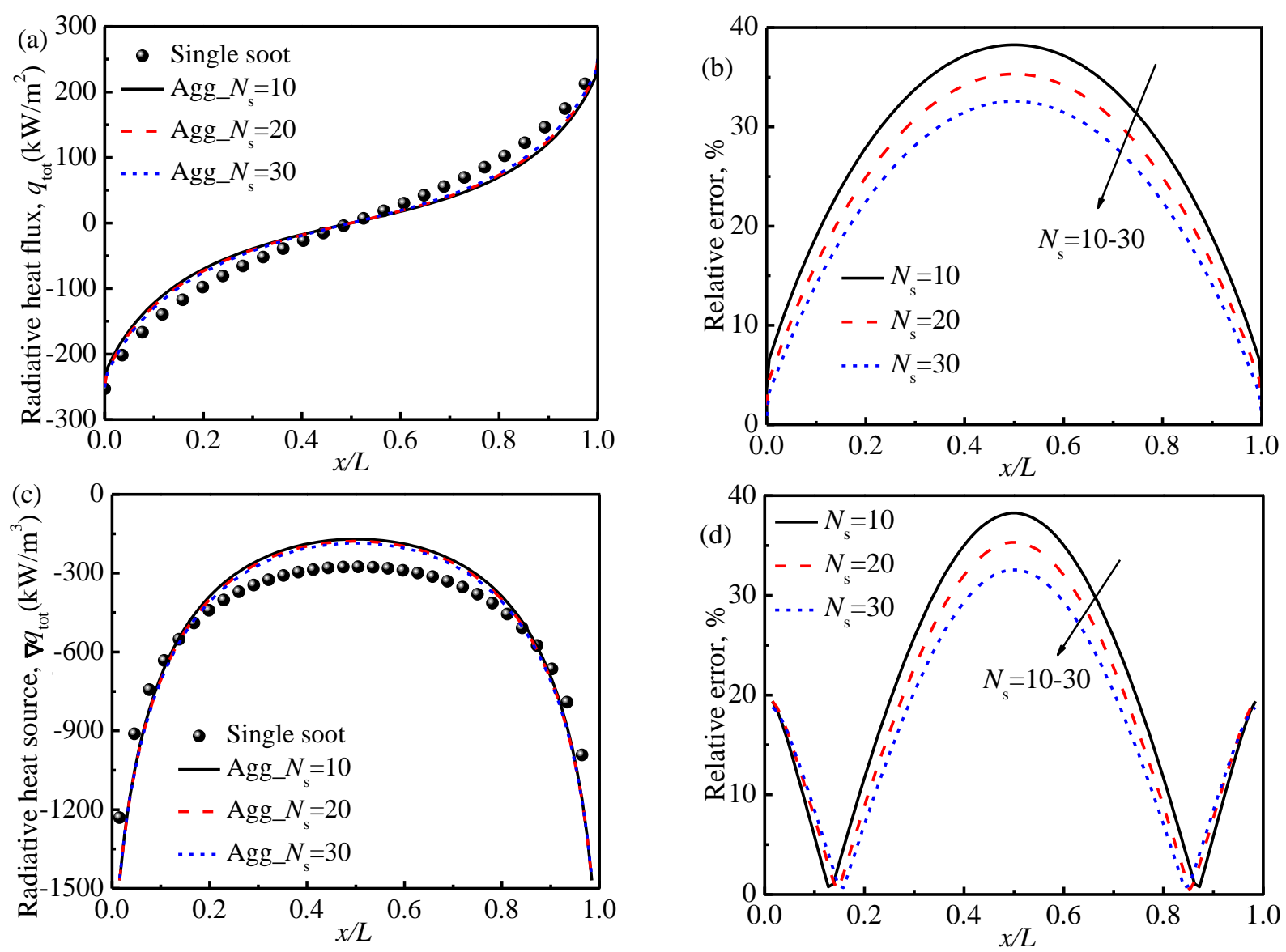

Fig. 11 Radiative heat transfer properties in the gas-soot mixture with different number of monomers in the aggregates: (a) and (b) denoting the value and corresponding relative error of radiative heat flux; (c) and (d) denoting those for radiative heat source.

\section{Conclusions}

Based on the full-spectrum k-distribution (FSK) method and Generalized Multiparticle Mie-solution (GMM) method, the effects of geometric feature parameters of soot aggregates on the radiative heat transfer properties in the 
gas-soot mixtures are investigated thoroughly. The results reveal that larger deviations between radiative heat transfer properties of the gas-soot mixtures with soot aggregates and those with single soot particles will be obtained when increasing the fractal dimension and radius of primary monomers or decreasing the total number of primary monomers in the aggregates. Soot aggregation plays an important role in studying the radiative heat transfer in the gas-soot mixtures and should be considered as much as possible to ensure the calculation accuracy of heat transfer simulation.

\section{Acknowledgments}

The supports of this work by the National Natural Science Foundation of China (No: 51806103), Jiangsu Provincial Natural Science Foundation (No: BK20170800), Open Funds of Aero-engine Thermal Environment and Structure Key Laboratory of Ministry of Industry and Information Technology (No. CEPE2018005), and they are gratefully acknowledged. The authors also would like to thank $\mathrm{Xu} \mathrm{Yu}$-lin, University of Florida, for providing the GMM program online. A very special acknowledgement is made to the editors and referees who make important comments to improve this paper.

\section{References}

Chang, H. and Charalampopoulos, T. T., Determination of the wavelength dependence of refractive indices of flame soot, Proceedings of the Royal Society A, Vol.430 (1990), pp.577-591.

Chu, H., Liu, F. and Consalvi, J. L., Relationship between the spectral line based weighted-sum-of-gray-gases model and the full spectrum k -distribution model, Journal of Quantitative Spectroscopy \& Radiative Transfer, Vol.143 (2014), pp.111-120.

Dannecker, R., Noll, B., Hase, M., Krebs, W., Schildmacher, K. U., Koch, R. and Aigner, M., Impact of radiation on the wall heat load at a test bench gas turbine combustion chamber: Measurements and cfd simulation, ASME Turbo Expo 2007: Power for Land, Sea and Air, (2007), pp.1311-1321.

Dorigon, L. J., Duciak, G., Brittes, R., Cassol, F., Galarça, M. and França, F. H. R., Wsgg correlations based on hitemp2010 for computation of thermal radiation in non-isothermal, non-homogeneous $\mathrm{H}_{2} \mathrm{O} / \mathrm{CO}_{2}$ mixtures, International Journal of Heat \& Mass Transfer, Vol.64 (2013), pp.863-873.

Gouyet, J.-F. and Mandelbrot, B., Physics and fractal structures, (1996), p.343, Masson Paris.

Guo, J., Hu, F., Luo, W., Li, P. and Liu, Z., A full spectrum k-distribution based non-gray radiative property model for fly ash particles, International Journal of Heat \& Mass Transfer, Vol.118 (2018), pp.103-115.

Guo, J., Li, X., Huang, X., Liu, Z. and Zheng, C., A full spectrum k-distribution based weighted-sum-of-gray-gases model for oxy-fuel combustion, International Journal of Heat \& Mass Transfer, Vol.90 (2015), pp.218-226.

Hofgren, H. and Sundén, B., Evaluation of planck mean coefficients for particle radiative properties in combustion environments, Heat \& Mass Transfer, Vol.51 (2015), pp.507-519.

Kangwanpongpan, T., França, F. H. R., Silva, R. C. D., Schneider, P. S. and Krautz, H. J., New correlations for the weighted-sum-of-gray-gases model in oxy-fuel conditions based on hitemp 2010 database, International Journal of Heat \& Mass Transfer, Vol.55 (2012), pp.7419-7433.

Kholghy, M. R., Afarin, Y., Sediako, A. D., Barba, J., Lapuerta, M., Chu, C., Weingarten, J., Borshanpour, B., Chernov, V. and Thomson, M. J., Comparison of multiple diagnostic techniques to study soot formation and morphology in a diffusion flame, Combustion \& Flame, Vol.176 (2017), pp.567-583.

Li, H., Liu, C., Bi, L., Yang, P. and Kattawar, G. W., Numerical accuracy of 'equivalent' spherical approximations for computing ensemble-averaged scattering properties of fractal soot aggregates, Journal of Quantitative Spectroscopy and Radiative Transfer, Vol.111 (2010), pp.2127-2132.

Li, S. N., Yuan, Y., Liu, B., Wang, F. Q. and Tan, H. P., Local error and its identification for microlens array in plenoptic camera, Optics and Lasers in Engineering, Vol.108 (2018), pp.41-53.

Li, S. N., Zhu, Y., Zhang, C., Yuan, Y. and Tan, H. P., Rectification of images distorted by microlens array errors in plenoptic cameras, Sensors, Vol.18, (2019), pp.1-10.

Li, T. J., Li, S. N., Yuan, Y., Wang, F. Q. and Tan, H. P., Light field imaging analysis of flame radiative properties based on monte carlo method, International Journal of Heat and Mass Transfer, Vol.119, (2018), pp.303-311.

Liu, L. and Mishchenko, M. I., Effects of aggregation on scattering and radiative properties of soot aerosols, Journal of geophysical research, Vol.110 (2005), D11211.

Liu, L., Mishchenko, M. I. and Patrick Arnott, W., A study of radiative properties of fractal soot aggregates using the superposition T-matrix method, Journal of Quantitative Spectroscopy and Radiative Transfer, Vol.09 (2008), pp.2656-2663.

Mackowski, D. W., A simplified model to predict the effects of aggregation on the absorption properties of soot 
particles, Journal of Quantitative Spectroscopy and Radiative Transfer, Vol.100 (2006), pp.237-249.

Meakin, P., Majid, I., Havlin, S. and Stanley, H. E., Topological properties of diffusion limited aggregation and cluster-cluster aggregation, Journal of Physics A: Mathematical and General, Vol.17 (1984), pp.975-987.

Mishchenko, M. I., Travis, L. D. and Lacis, A. A., Scattering, absorption, and emission of light by small particles (2002), p.68, Cambridge university press.

Modest, M. F., Radiative heat transfer, (2013), p.499, McGraw-Hill New York.

Qi, H., Zhao, F. Z., Ren, Y. T., Qiao, Y. B., Wei, L. Y., Islam, M. A. and Ruan, L. M., Experimental research on noninvasive reconstruction of optical parameter fields based on transient radiative transfer equation for diagnosis applications, Journal of Quantitative Spectroscopy and Radiative Transfer, Vol.222, (2019), pp.1-11.

Ren, Y. T., Chen, Q., Qi, H., Ruan, L. M. and Dai, J. M., Phase transition induced by localized surface plasmon resonance of nanoparticle assemblies, International Journal of Heat and Mass Transfer, Vol.127, (2018), pp.244-252.

Rothman, L. S., Gordon, I. E., Barber, R. J., Dothe, H., Gamache, R. R., Goldman, A., Perevalov, V. I., Tashkun, S. A. and Tennyson, J., "Hitemp, the high-temperature molecular spectroscopic database", Journal of Quantitative Spectroscopy \& Radiative Transfer 111, 2139 (2010).

Sorensen, C. M., Yon, J., Liu, F., Maughan, J., Heinson, W. R. and Berg, M. J., Light scattering and absorption by fractal aggregates including soot, Journal of Quantitative Spectroscopy \& Radiative Transfer Vol. 217 (2018), pp. 459-473.

Sun, S., Qi, H., Zhao, F., Ruan, L. M. and Li, B., Inverse geometry design of two-dimensional complex radiative enclosures using krill herd optimization algorithm, Applied Thermal Engineering, Vol.98 (2016), pp.1104-1115.

Tashkun, S. A., Perevalov, V. I., Teffo, J. L., Bykov, A. D. and Lavrentieva, N. N., CDSD-1000, the high-temperature carbon dioxide spectroscopic databank, Journal of Quantitative Spectroscopy \& Radiative Transfer, Vol.82 (2003), pp.165-196.

Trivic, D. N., 3D radiation modeling of nongray gases-particles mixture by two different numerical methods, International Journal of Heat \& Mass Transfer, Vol.70 (2014), pp.298-312.

Wang, C., Modest, M. F. and He, B., Full-spectrum k-distribution look-up table for nonhomogeneous gas-soot mixtures, Journal of Quantitative Spectroscopy \& Radiative Transfer, Vol.176 (2016), pp.129-136.

$\mathrm{Xu}, \mathrm{Y}$. 1. and Khlebtsov, N. G., Orientation-averaged radiative properties of an arbitrary configuration of scatterers, Journal of Quantitative Spectroscopy and Radiative Transfer, Vol.79 (2003), pp.1121-1137. 